Putting

\section{song to use}

\section{John Krebs}

Acoustic Communication in Birds. Vol.1. Production, Perception and Design

Features of Sounds. Vol.2. Song

Learning and Its Consequences.

Edited by Donald K. Kroodsma,

Edward H. Miller and Henri Ouellet.

Academic: 1983. Vol.1. Pp.368. Vol.2.

Pp.387. Vol.1. \$36, £23.80; Vol.2.

$\$ 39$, f25.80.

NAOMI Mitchison once illustrated the liberated biological atmosphere of her family background by referring to early encounters with scientists whose lives were devoted to the study of subjects "as irrelevant as bird song". An alternative view comes from R.A. Hinde's introduction to these two volumes: "It is probably true to say that the study of bird song has done as much for the advancement of ethology as the study of any other specific aspect of behaviour"'

The many ramifications and wideranging influence of bird song research is well illustrated in this collection of 17 original articles. Areas in which songresearchers are contributing important work include neuroanatomy and neuroendocrinology, genetic differentiation of natural populations, sensory perception, sexual selection, and learning. As impressive as the diversity of song research, is the way which common themes link together different approaches. For example, the "auditory template hypothesis" originally proposed by P. Marler (to whom the two volumes are appropriately dedicated) and his associates to account for results in studies of song development, is discussed not only in this context (by Kroodsma), but also in other chapters. It is referred to by Arnold as a starting point for neuroanatomical investigations of pathways of auditory feedback to 'song centres' in the brain and by Morton in a discussion of how it is that birds are able to assess the degree of degradation of sound as a result of its passage through the environment.

The role of song in mate choice is another recurrent theme - discussed in relation to maintenance of genetic isolation between populations at song dialect boundaries (Baker), in the context of the evolution of song complexity through sexual selection (Catchpole) and in accounting for the enlargement of the hyperstriatum ventrale which is associated with the acquisition of a large song repertoire in canaries and marsh wrens.

It is not only in bringing home these common themes that the book does a good job. Most of the individual chapters include thorough surveys with useful tables of literature on song learning, vocal mimicry, auditory sensitivity, song dialects, duetting and so on. It is hard to single out individual chapters for special praise since at least half are excellent. One general impression emerged: work on neura ${ }^{1}$ control, perception, motor produr.ton and physical aspects of transmission of bird sounds has made more progress than research on their functional significance - what they actually mean to both the birds that utter them and the birds that hear them.

Although various facets of vocal communication have been thoroughly documented for many years - vocal mimicry, song dialects, elaborate song repertoires of individual males, matched counter-singing between rivals, and so on - understanding their biological significance (or even understanding whether it is worth asking about their significance) seems to have progressed relatively little in the last decade or so. I am not sure why this is, but offer two suggestions. First, little successful thought seems to have been given to developing new techniques for answering questions about the biological significance of bird sounds; indeed much recent work still consists of description, correlation and

\section{Modelling nuclei}

\section{P.E. Hodgson}

\section{Statistical Theory and Random}

Matrices.

By Moshe Carmeli.

Marcel Dekker: 1983. Pp.203. \$35.

THE properties of individual low-energy nuclear states can be determined by nuclear reaction studies, and they can often be quite well understood using various nuclear models. At higher excitation energies the density of states becomes so large that it is impossible to study them individually. Instead, statistical techniques can be applied to define and extract useful information. Professor Moshe Carmeli provides a clear, terse and authoritative introduction to the methods used to do this.

The most important statistical properties are the density of levels and the distributions of their widths, spacings and angular momenta, together with the energy variations of all these quantities. These define the properties of nuclei at high excitations, and also provide the essential data for calculating the cross-sections of many high-energy reactions. Many experimental studies of the statistical properties of nuclei have been made, and these require theoretical explanation.

As Professor Carmeli explains in his introductory chapter, we can understand the statistical properties of nuclei if we renounce any knowledge of their detailed behaviour and see if their properties can be deduced from some very general assumptions. In ordinary statistical mechanics we interpretation with informed guesswork rather than incisive experiments. Second, perhaps the functional questions cannot be fully answered until more is known about the mechanisms of sound production and perception. To take a simple example, discussions of the significance of elaborate song repertoires are currently going on in the absence of any information abut how the birds themselves perceive complex sounds, whether they categorize and differentiate them in the same way as we do. Once there is a good base of information about categorical perception, it may be possible to view the question of functional significance in a new light.

Johannes Kepler wrote in his Mysterium cosmographicum "We do not ask for what purpose the birds do sing, for song is their pleasure since they were created for singing". While there has been progress since Kepler's day, there is still some way to go before we will know exactly why birds sing the way they do.

John Krebs is Lecturer in Zoology at the Edward Grey Institute, University of Oxford and Fellow of Pembroke College.

assume that all the states of a very large ensemble are equally probable, but a different approach must be used to derive the statistics of nuclear levels. Instead of renouncing exact knowledge of the state of the system, we renounce knowledge of the nature of the system itself. A nucleus is considered to be a system of many particles interacting in an unknown way. The statistical mechanics of such ensembles of systems in which all possible laws of interaction are equally probable was first developed by Wigner, and has been extensively applied to analyse nuclear data since then.

If the interaction is known, the energy levels are obtained as the eigenvalues of a matrix. The statistical theory is thus concerned with the properties of states obtained by diagonalizing matrices with elements following postulated distribution laws. The mathematical theory of such distributions, and their implications, forms the core of Professor Carmeli's book. He shows how quite simple distribution functions give states whose statistical properties are in good accord with the experimental data.

The main emphasis is on the mathematics of the connections between statistical theory and random matrices; in addition comparisons are made with experimental data following the recent review of Brody and colleagues. Extensive references to the original literature are provided, together with mathematical appendices on multivariate distributions and the ergodic properties of random matrices.

P.E. Hodgson is Head of the Nuclear Physics Theoretical group at the Nuclear Physics Laboratory, University of Oxford. 